\title{
Multi-person Virtual Rehearsal System Based on Optical Location Tracking
}

\author{
Dongjin Huang \\ Shanghai Film Academy \\ Shanghai University \\ Shanghai, China \\ djhuang@shu.edu.cn
}

\author{
Hejuan Li \\ Shanghai Film Academy \\ Shanghai University \\ Shanghai, China \\ 1575037745@qq.com
}

\author{
Youdong Ding \\ Shanghai Film Academy \\ Shanghai University \\ Shanghai, China \\ ydding@shu.edu.cn
}

\author{
Wen Tang \\ Department of Creative Technology \\ University of Bournemouth \\ Fern Barrow, United Kingdom \\ wtang@bournemouth.ac.uk
}

\author{
Houchao $\mathrm{Zu}$ \\ Department of Technology \\ Shanghai ChingMu technology co. LTD \\ Shanghai, China \\ zuhouchao@chingmu.com
}

\author{
Haiwei Zhang \\ Department of Technology \\ Shanghai ChingMu Tech. co. LTD \\ Shanghai, China \\ zhanghaiwei@chingmu.com
}

\begin{abstract}
Rehearsal system is the latest form of 3D technology applications in the field of film and television. In this paper, we propose a novel multi-person virtual rehearsal system based on passive optical motion capture technology. A new fast location algorithm for human motion gesture is presented, which combines the traditional forward kinematic algorithm with the location tracking algorithm of markers, and uses noise elimination filter method and Euclidean distance to stability and evaluation of markers data. The experimental result shows that this system, focusing on the interaction of actors and scenes, lights, cameras, etc, could realize the shooting test and scene rehearsal of VR films well with high capture precision, tracking stability and real-time performance, and improving the efficiency and quality of VR films greatly.
\end{abstract}

Keywords-virtual reality; rehearsal system; motion capture

\section{INTRODUCTION}

Rehearsal system is mentioned to the preparatory exercises before the large-scale implementation of a certain operation. The advances in science and technology have pushed the rehearsal system towards the direction of virtualization with more clear 3D effect. At present, virtual rehearsal is generally applied in education [1], sports [2] and medical [3]. However, in VR films is rarely used. In contrast to traditional movies, VR films have a long shooting cycle, difficult shooting and high production costs, and proposing the virtual rehearsal system is very necessary.

Motion capture, as the important technology of virtual rehearsal system, is the process of measuring, tracking, and recording motion trajectories of objects in 3D space. Mainly by the sensors, signal capture devices, data transmission equipment and data processing equipment, which can be divided into mechanical, optical, magnetic, acoustic and inertial trackers. Currently, motion capture technology has been widely used. B Taetz et al [4] used inherent motion capture system to track human motion, Zhang [5] and Shafaei A et al [6] selected depth-camera. However, both inertial motion tracking and depth-camera have inherent drawbacks which cant be eliminated theoretically and experimentally.

In order to solve the above problems, this paper presents a multi-person virtual rehearsal system based on passive optical technology for VR films production. We use multiple near-infrared high-sensitivity dedicated cameras to track actors performance, and restore the role of squat, hug, and other complex action with better tracking stability. By Unity3D platform, real-time interaction among actors and virtual scenes, cameras, lighting is achieved to get through the pre-shot and post-production channels.

\section{Algorithm And System}

\section{A. System overview}

The approximately process of our system is shown in Figure 1. Firstly, the passive optical motion capture equipment is selected to track actors performance, and human motion gesture is located and replayed in real time. Then, the system uses motion builder and noise elimination filter to require and ensure data stability, selecting the Euclidean distance to evaluate it effectively. Immediately following, with the Unity3D, we produces virtual rehearsal through virtual scene, virtual camera and virtual illumination. Finally, the rehearsal clips and parameters of camera, light, etc are output for preshot and post-production.

\section{B. Fast Location Algorithm for Human Motion Gesture}

The Optical motion capture algorithm flowchart shown in Figure 2. Based on the forward kinematic algorithm, we propose a fast location algorithm for human motion posture to achieve the markers high-precision location, 3D reconstruction and tracking detection. With the noise elimination filter algorithm, an improved detection method is proposed to ensure the stability of tracking. The Euclidean distance is used to measure errors by average distance between the estimated value and true value of the three-dimensional 


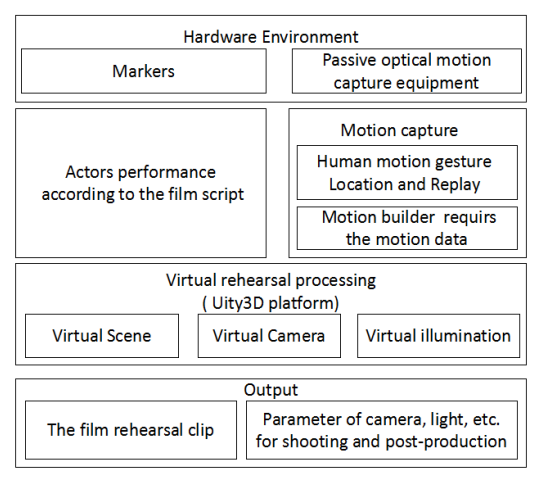

Figure 1. System architecture

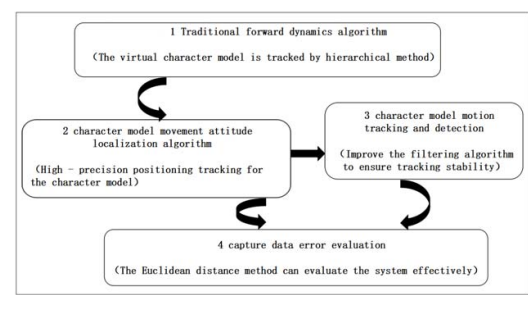

Figure 2. Optical motion capture algorithm flowchart

position of the joint of the human body model, that could make effective evaluation of the system.

\section{RESUlTS AND DISCUSSION}

Our system runs on Intel (R) Core (TM) i7-6500 4.00GB CPU@2.50GHz memory machine. And we select 4 actors and 16 passive optical cameras to capture their performance, build a virtual military scene by Unity3D and preview a military theme through Head Mount Display. The result of experiment is shown in Figure 3.

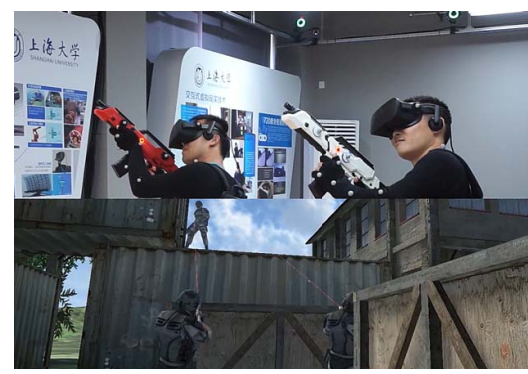

Figure 3. Multi-person military film rehearsal

During the whole process of rehearsal, the system captures the actors' motion with stable performance and low latency, as shown in Figure 4. The whole data is divided into two stages: walking and jogging. The data line fluctuates relatively gentle in the early stage, but it fluctuates greatly during the jogging phase. Overall, the different motion of actions in our system can be better demonstrated and the whole data line is pretty smooth and stable.

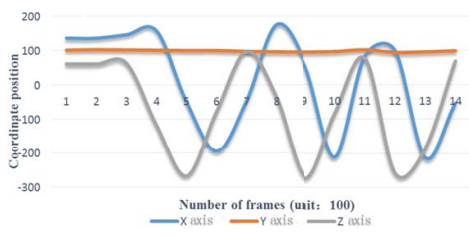

Figure 4. Tracking stability test result

\section{Conclusion}

In this paper, we propose the virtual rehearsal system for VR films. The fast optical location algorithm can effectively solve the limits of blocking markers, unstable tracking and poor interaction etc. And the captured data from our system can be applied into pre-production and post production of VR films, greatly reducing the production costs, enhancing the interactivity and improving the production efficiency and quality.

\section{ACKNOWLEDGMENT}

This work was supported by NSFC of China(61402278), the Shanghai Committee of Science and Technology(16511101302), Construction Program of Shanghai Engineering Research Center(16dz2251300), and Shanghai University the peak discipline of filmology.

\section{REFERENCES}

[1] Barmaki R and Hughes C E. Providing real-time feedback for student teachers in a virtual rehearsal environment. ACM International Conference on Multimodal Interaction, 531-537, 2015.

[2] Gesan LIU, Jianjun ZHAO, and Yunhui GUO. Research and design of real time interactive preview platform for motion control system in movie virtual production. Advanced Motion Picture Technology, 4:20-30, 2017.

[3] Park S H, Lee J, Jeong J W, and et al. Description language for graph-based representation in surgical rehearsal. International Conference on Systems and Informatics, 922-926, 2015.

[4] Taetz B, Bleser G, Miezal M. Towards self-calibrating inertial body motion capture. International Conference on Information Fusion, 2016.

[5] Chang Zhang, Lin $\mathrm{Xu}$, and Guangyi Sun. Motion capture system based on rgb-d camera and imu data fusion. Journal of systems simulation, 27(10):25822588, 2015.

[6] Shafaei A and Little J J. Real-time human motion capture with multiple depth cameras. 13th Conference on Computer and Robot Vision, 24-31, 2016. 